\title{
Spine Degeneration and Inflammation
}

\author{
David J. Wilson and Marcelo de Abreu
}

\section{Learning Objectives}

- To understand how degenerative disorders of the spine develop and exhibit themselves on imaging.

- To understand how inflammatory disorders of the spine develop and exhibit themselves on imaging.

- To recognize inflammatory and degenerative disorders in the differential diagnosis of spinal disease.

\subsection{Degenerative Diseases of the Spine}

At some point in our lives, almost all of us will suffer from back pain. The clear majority will have degenerative disease of the discs or facet joints. A small minority will have tumours, insufficiency fractures, inflammatory joint disease or infection. Differentiation of these conditions is clearly critical for management. In this syllabus, we will cover degeneration and inflammatory joint disease and describe how imaging and image-guided intervention are important in precise diagnosis and planning therapy. Brief mention will be made of the alternative diagnoses.

\subsubsection{Anatomy}

The spine consists of segments comprising a vertebral body, articulations with adjacent vertebrae and in the thoracic region with ribs. The sacrum is a specialized segment where vertebrae are embryologically fused and the whole structure acts as a part of the pelvic ring. These articulations are at risk from degenerative and inflammatory disorders.

\section{J. Wilson $(\bowtie)$}

St Luke's Radiology Oxford Ltd., Oxford, UK

e-mail: david@ @lro.co.uk

\section{M. de Abreu}

Radiology, Hospital Mãe de Deus, Porto Alegre, RS, Brazil
Ligaments and muscles bridge vertebral segments and allow flexion, extension, tilt and rotation. It is useful to think of a mechanical segment as comprising the vertebra and its attachments.

The intervertebral disc's function is to allow the above movements at the same time as maintaining transverse stability. The disc sits at the division of a mechanical segment and is subject to considerable physical load. The joints between vertebrae must slide and rotate to allow movement. The annulus fibrosis is bound to the edge of the vertebral body and comprises 15-25 concentric layers. Within the disc, the nucleus pulposus is soft and very hydrated. It transmits hydrostatic pressure and acts as a buffer between the end plates.

The spine will experience compression, tensile force, shear force, bending movement and torsional movement. Normal function of joints, ligaments and muscle spreads these loads across many segments. However, when disease intervenes, focal load will increase at adjacent segments which must move more dramatically to compensate for less movement at diseased levels. It is therefore common that symptoms in one part of the spine precipitate pain and dysfunction throughout the vertebral column.

We should not consider the vertebral body to be a simple building block. It has a trabecular internal structure which responds to load applied in different ways. The discs distribute load across the vertebral body, and when they become degenerate, point pressures may become very much higher than in the young person's spine. In older patients, the bone mineral quantity is reduced often considerably, and these two processes combine to cause catastrophic collapse presenting as insufficiency fracture. The combination of osteoporosis and degenerative disease with mechanical dysfunction are major factors in this very common disorder.

Ligamentous attachments in the spine are extensive and complex. Some ligaments restrain the spinal segment from excessive motion, but others provide integral stability binding vertebra to disc, most particularly the posterior and anterior longitudinal ligaments. It is easy to underestimate the 
complexity of the ligamentous structures around the sacroiliac region; there are anterior and posterior complexes with deep and superficial layers posteriorly. These envelop the articulation of the sacrum to the pelvis. The sacroiliac joint has both fibrous and synovial components and is therefore at risk in patients with systemic inflammatory disease of joints.

This concept of mechanical activity is very important when considering disease; it is easy to view the spine as a solid static structure, but observation and the above descriptions should make it clear that movement is a primary function and the one that is most frequently lost in patients who present with pain and dysfunction. Imaging is currently based on a static description of morphology, and abnormalities of function are more difficult to determine [1] (Figs. 14.1 and 14.2).

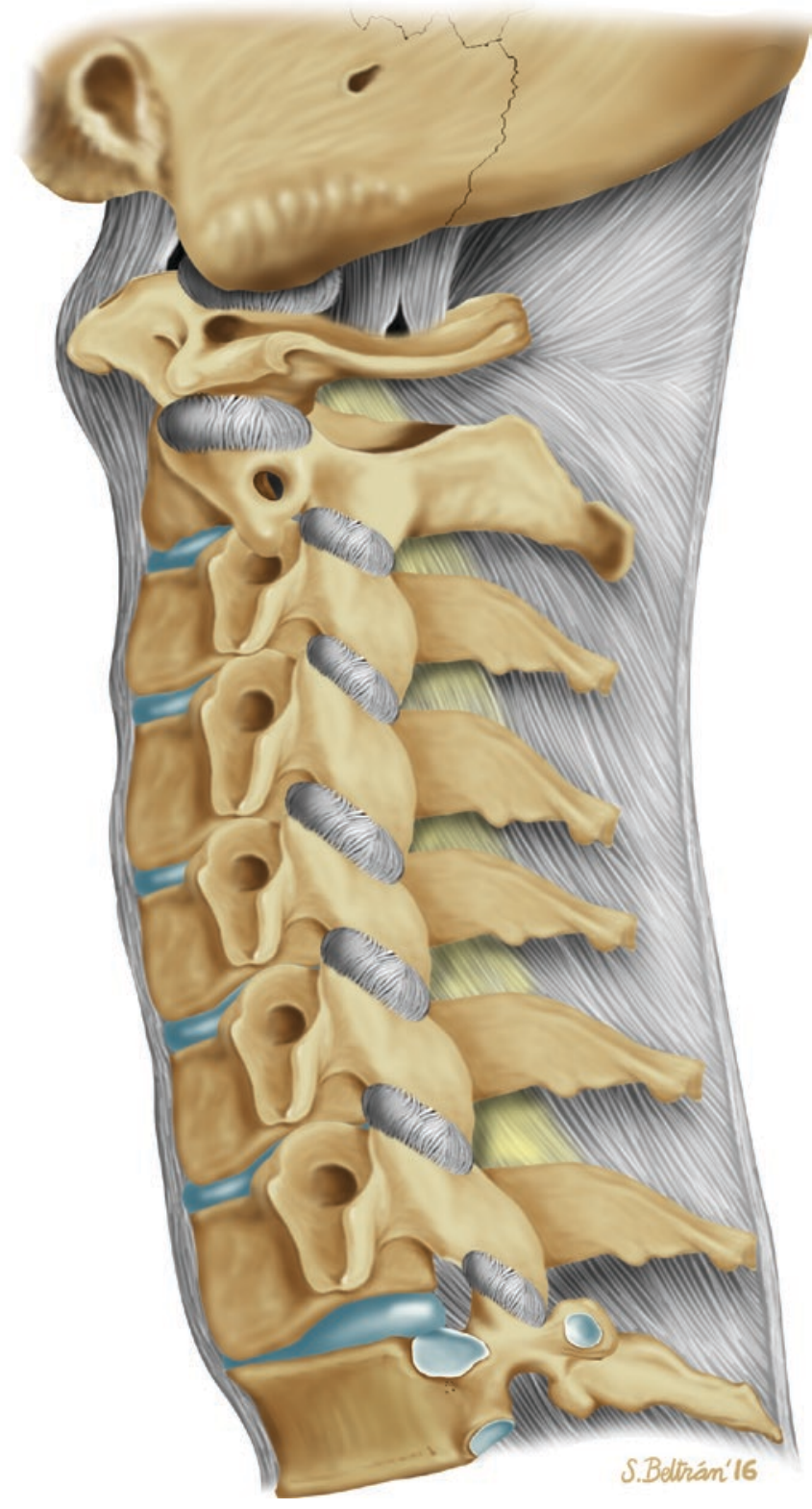

Fig. 14.1 Diagram of morphology of the vertebral supports
Key Point

- The static anatomy must be understood in detail, and those interpreting images must recognize that the spine is a flexible and movable structure, and conventional imaging does not include dynamic studies.

\subsubsection{Degenerative Disease}

The degenerative process seems to be an inevitable consequence of ageing. The changes may be observed in the third decade and progress throughout life. The mechanisms are:

1. Relatively poor blood supply to the intervertebral discs

2. Multiple micro injuries to discs

3. Cartilage wear in the joints

4. Reduction in the number of chondrocytes

5. Vascular ingrowth into the disc

6. Marginal osteophytes on vertebral bodies and joints

7. Herniation of discs

8. Osteopenia and osteoporosis

9. Biomechanical overload due to disease at other segments

There is a genetic element to the speed of progress of degeneration probably related to the proteoglycan binding of collagen fibres. More linkages mean stiffer collagen which is more brittle; fewer linkages mean softer collagen which is more likely to tear and displace.

The microtrauma element of degeneration is accelerated by impact and multiple minor traumatic incidents and therefore has an occupational and sporting association.

\subsubsection{Manifestations of Degenerative Disease}

\subsubsection{Discs}

Small tears within the disc lead to annular tears. A tear may be seen as a defect in the annulus, containing soft tissue material which may take on an increased water content. There is considerable debate as to whether these tears with high signal material are a cause of localized back pain [24]. Tears with scar tissue and repair produce low MRI signal. There is also considerable debate over alterations in water content in the annulus; some regard this as the main cause of reduced MRI signal on all sequences, but others believe that scar tissue from microtrauma is a major element. The consequence of all these changes is reduction in pliability and tension within the annulus and a tendency for disc height reduction. This in turn leads to buckling of the longitudinal ligaments. Less commonly described in imag- 


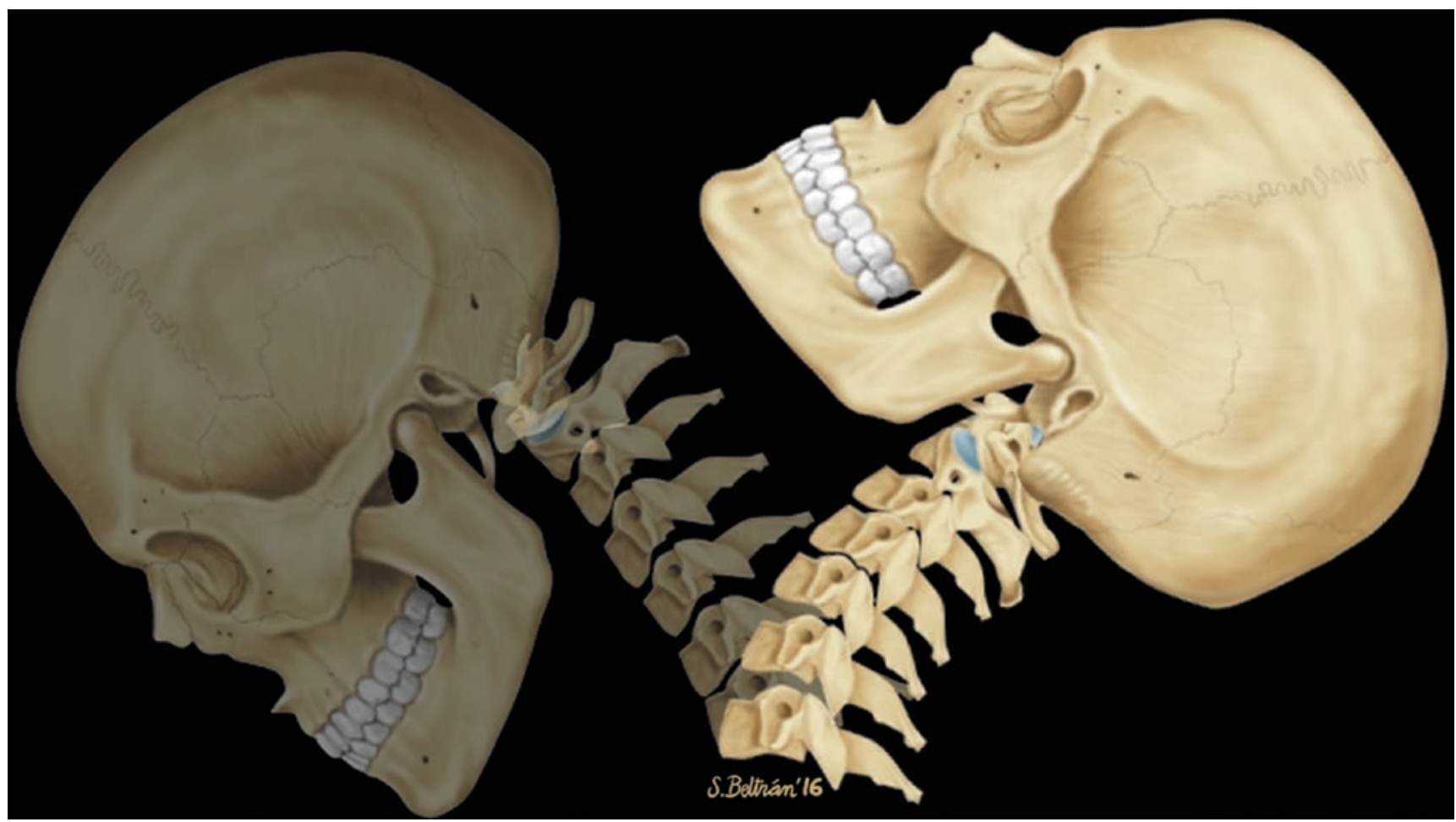

Fig. 14.2 Diagram to demonstrate spine movement

ing interpretation but probably a much more important factor in morbidity is mechanical instability due to loss of tension within the annulus and laxity of the margins of the disc. Frank herniation of disc material through tears in the annulus fibrosis may not only compress adjacent nerve roots but also releases several chemical irritants including prostaglandin, tumour necrosis factor alpha (TNF $\alpha$ ) and interleukins [5]. These chemicals may be part of the localized pain response and are potentially toxic to the adjacent nerves.

The loss of height in an intervertebral disc will in turn create mechanical instability, and this will lead to osteophytes that may impinge on adjacent nerves [6] (Fig. 14.3).

\subsubsection{Facet Joints}

As for all synovial joints, articular cartilage wear and mechanical instability lead to osteoarthritis with localized pain and osteophytes. New bone formation around the joint may considerably limit movement which in turn places additional load on adjacent segments. As degeneration is inevitably widespread and extremely common, it may be very difficult to determine the origin of pain as the presence of facet joint arthropathy does not predict that pain arises from this area. Synovial cysts may arise from the joint and may compress nerve roots. Haemorrhage into the cysts may increase the pressure. The fundamental problem is that facet joint disease is very common, and we have no imaging technique that scans for the origin of pain. The presence of facet

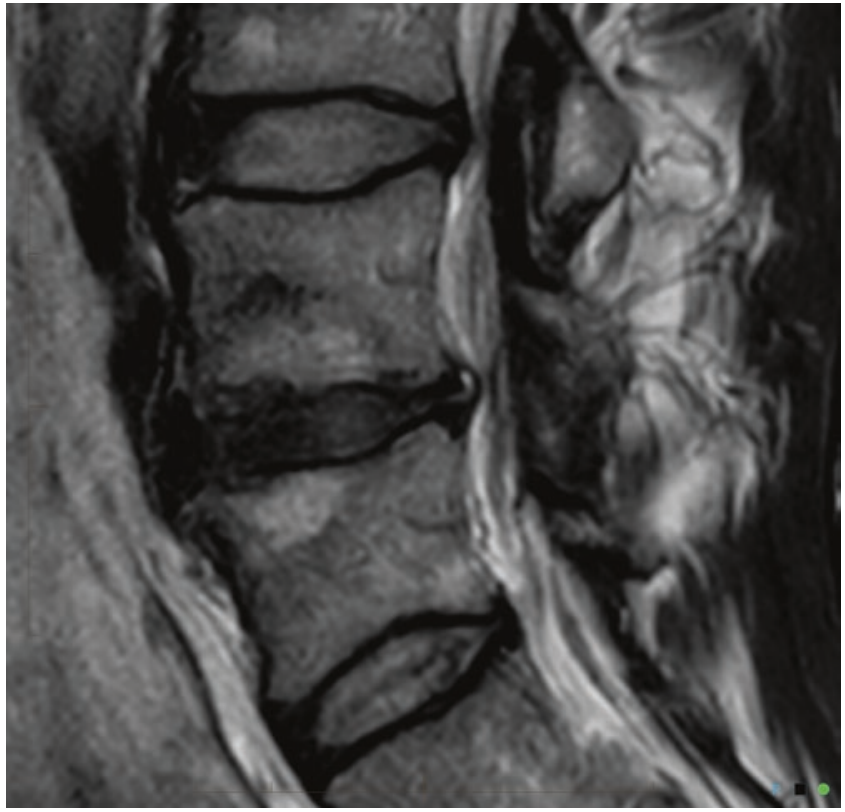

Fig. 14.3 MRI of annular tear

joint changes does not necessarily indicate an origin of pain; similarly, joints that look relatively normal may be the source of symptoms. Systems are closely interrelated, and there is association between muscle atrophy and facet joint disease; it may be that spine instability is exacerbated by this connection [7] (Figs. 14.4 and 14.5). 


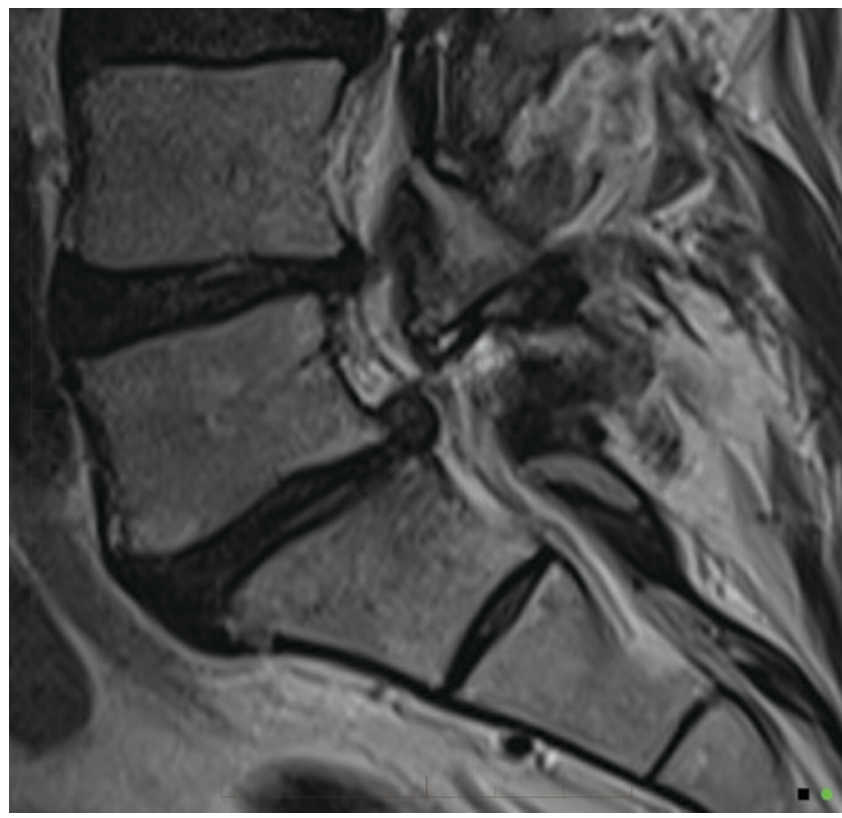

Fig. 14.4 Facet joint arthritis with a synovial cyst, disc prolapse and grade 1 spondylolisthesis leading to spinal stenosis

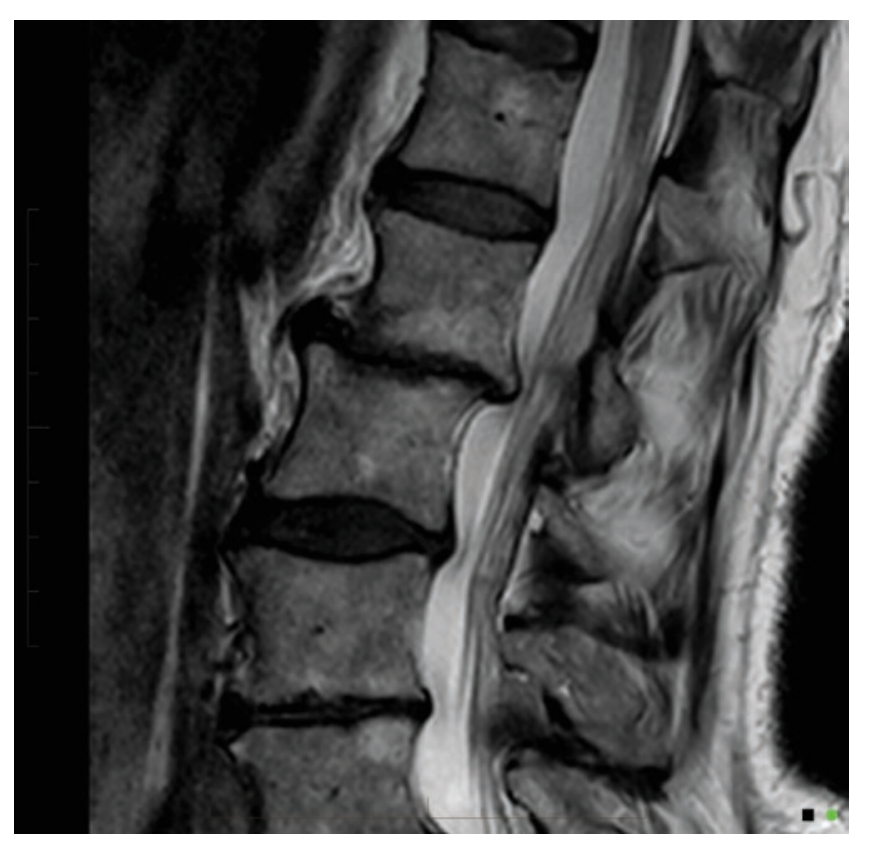

Fig. 14.5 Severe facet joint arthrosis with resultant instability and retrolisthesis of L1 and L2 beginning to impinge on the cauda equina. This image was taken in the supine position, and the changes will be worse when the patient stands

\subsubsection{End Plates}

Scheuermann (1877-1960) a Danish surgeon serving in Copenhagen in 1921 described a condition that he called osteochondritis deformans [8]. It comprises a deformity of the end plates, presenting in adolescence, which is now recognized to be a genetic autosomal dominant end plate dys- plasia [9]. The main candidate genes are COL2A1 and COL9A3. It is sometimes called osteochondrosis. It causes irregularity of the end plates which become prominent when the vertebral body reaches sufficient maturity, normally in adolescence. It is uncommon but a potential cause of thoracic pain. This may be a factor in later facet joint arthritis and segmental instability [10] (Fig. 14.6).

\subsubsection{Pars Interarticularis}

Degenerative disorders are uncommon in young people. However, it might be argued that stress fractures are a form of degeneration. They are caused by repetitive mechanical stress. Pars interarticularis stress fractures are common in young people. Typically, they occur in adolescents who are undertaking sport or physical activity that places repeated stress on bone which is not yet mature. MRI demonstrates bone oedema for some weeks before a stress fracture occurs. Whilst this is easy to identify in an MR image, it does require that the observer look specifically at the pars interarticularis for signal change on either a STIR sequence or a T2-weighted fat-suppressed sequence (STIR sequences are more sensitive). This is an important diagnosis to make because even a few days of additional activity may irretrievably fracture the bone. This can lead to a lifetime of back pain and disability. Strict exercise limitation for 6 weeks followed by a careful rehabilitation can lead to complete and permanent healing of the stress response (Figs. 14.7 and 14.8).

Stress of the end plate may lead to the changes described by Modic et al. which may be characterized by their MRI signal as bone oedema, fatty infiltration and sclerosis. There is some evidence that end plate stress changes particularly those with oedema are linked to back pain [11, 12], but there is poor evidence to show that lumbar surgery results are related to the stage of end plate change.

\subsubsection{Spinal Stenosis}

Individuals are born with spinal canals of differing dimension. Those with narrow canals need very little disc protrusion or osteophytes formation to cause pressure on the cord or cauda equina. Spinal stenosis is when the spinal canal is rendered too narrow due to disc or joint disease for normal blood supply to reach the neural structures. Patients will typically complain of back and leg pain which comes on after walking (spinal claudication, named after the Emperor Claudius of Rome who had walking difficulties); this eases after several minutes' rest. The disease is progressive and rarely undergoes spontaneous resolution. Therefore, surgery is an important treatment method, and imaging is essential in plotting the extent and severity. There is considerable literature on the efficacy of surgery, but a recent Cochrane review suggested that fusion plus decompression was no better than conventional decompression alone [13] (Fig. 14.9). 


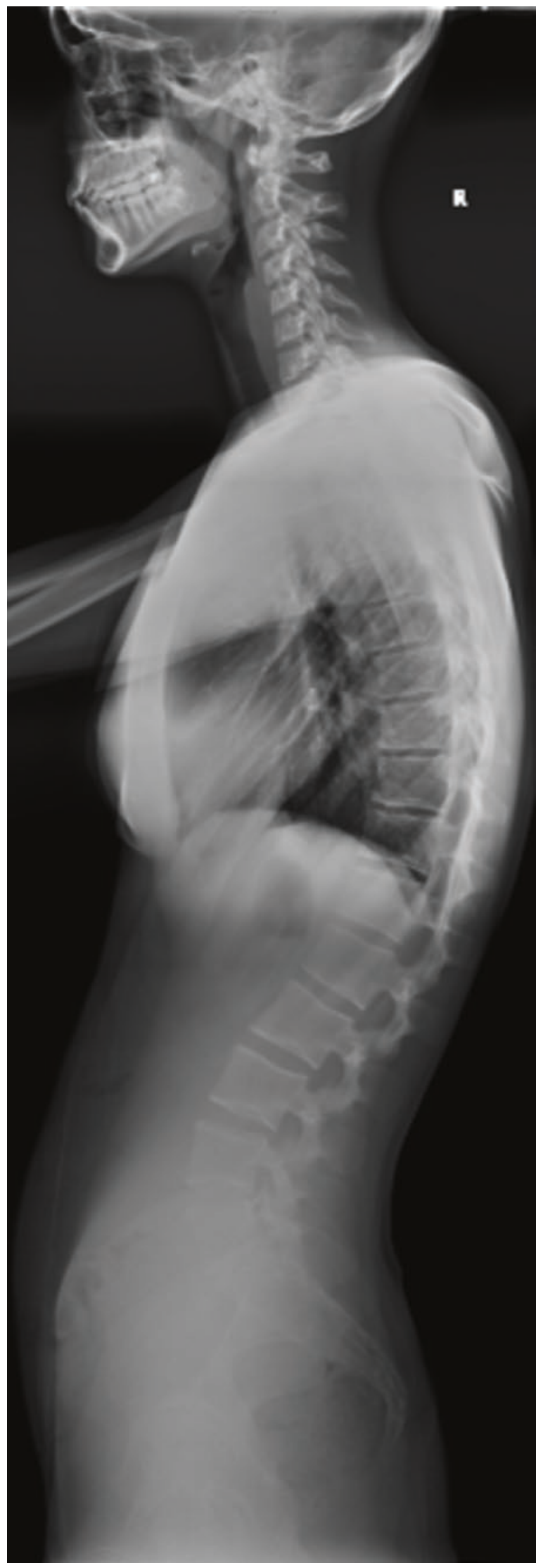

Fig. 14.6 Scheuermann's disease with irregular endplates in the lower thoracic spine
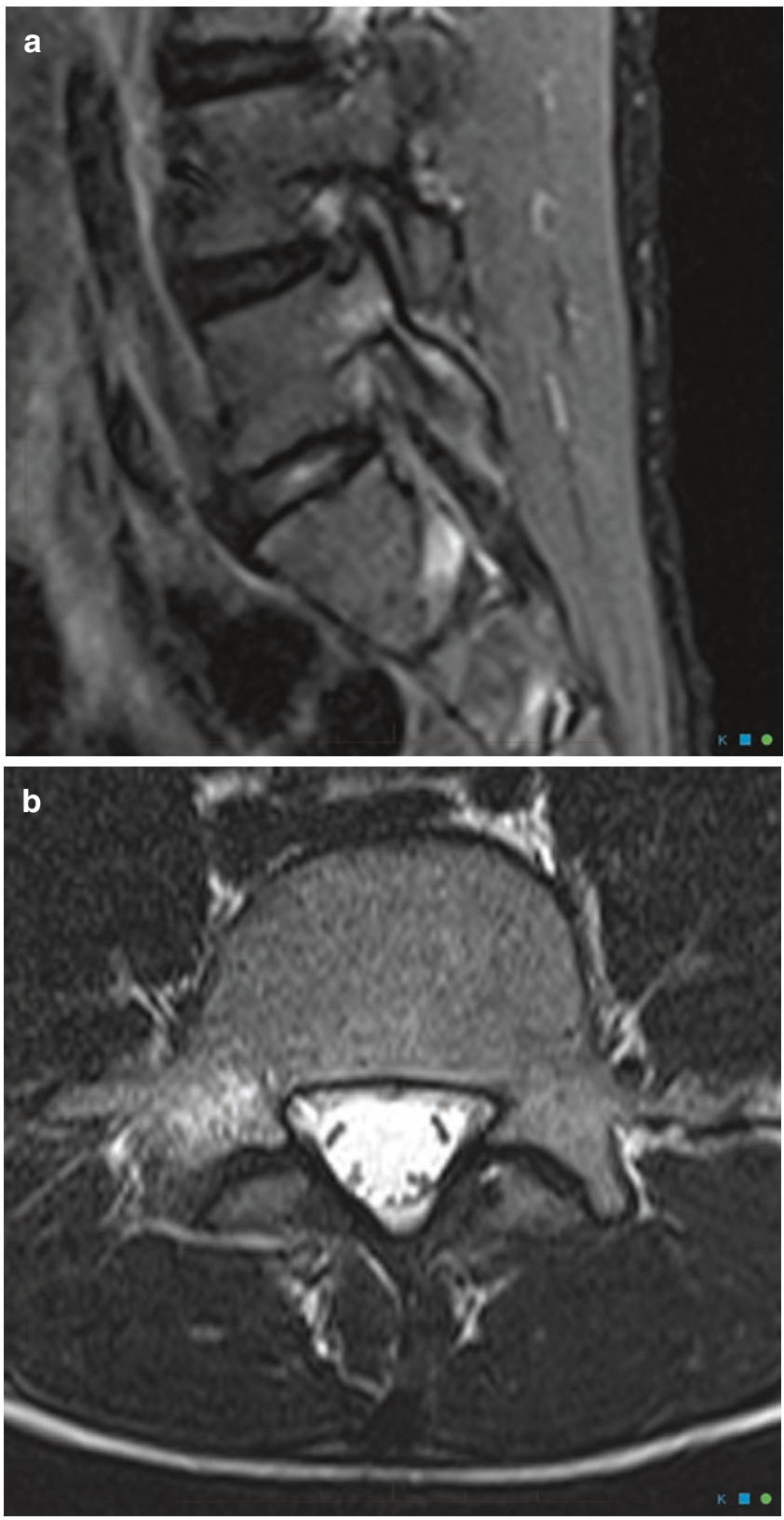

Fig. 14.7 (a, b) Unilateral stress response in the right pars interarticularis in a 15-year-old athlete

\subsubsection{Imaging}

\subsubsection{Conventional Radiographs}

Conventional radiographs of the spine have a principal role in assessing patients with trauma. This includes patients suspected of insufficiency fracture. However, it is now well recognized that they have limited if any value in the assessment of patients with back pain and are of minimal value in judging whether degenerative changes are affecting the patient in a way that might require interventional techniques. The appearances of advanced anky- 


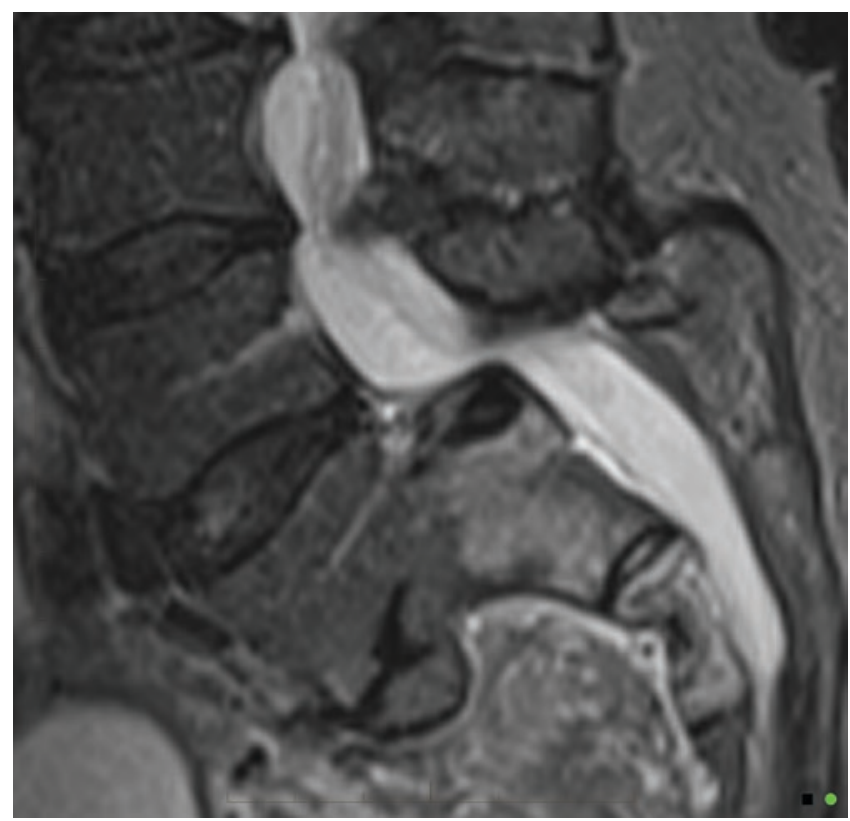

Fig. 14.8 Severe spondylolisthesis with surgical fusion. This is what happens when we miss stress response in teenagers

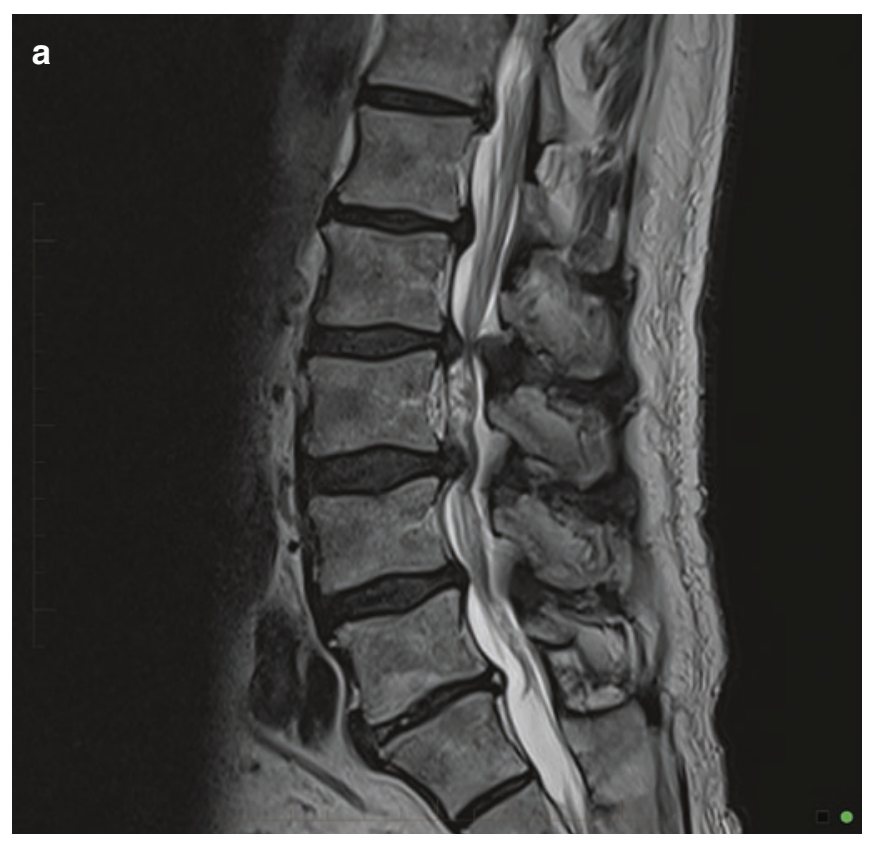

Fig. 14.9 (a) MRI spinal stenosis. Disc disease and facet joint hypertrophy lead to severe spinal canal stenosis at L2/L3 and moderate spinal stenosis at L3/L4. (b) Shows that the CSF has been completely effaced losing spondylitis are typical, but it might be argued that if you are trying to assess the disease at this stage, you are far too late. National guidance of the United Kingdom now states that conventional radiographs are not indicated for the investigation of back pain.

\subsubsection{Computed Tomography}

$\mathrm{CT}$ is the ideal method of assessing complex fractures and detecting destructive tumours. It will not demonstrate inflammatory changes at the early stage when they would be best treated, and it will not show bone oedema. It is much less effective than MRI at judging nerve root compression but can be used as an alternative when MRI is impossible.

\subsubsection{Magnetic Resonance Imaging}

MRI is the workhorse for the assessment and diagnosis of spinal disease. It is the preferred imaging for patients with back pain that fails to respond to conservative measures and those patients who have adverse clinical features or signs that might suggest infection, tumour or insufficiency frac-

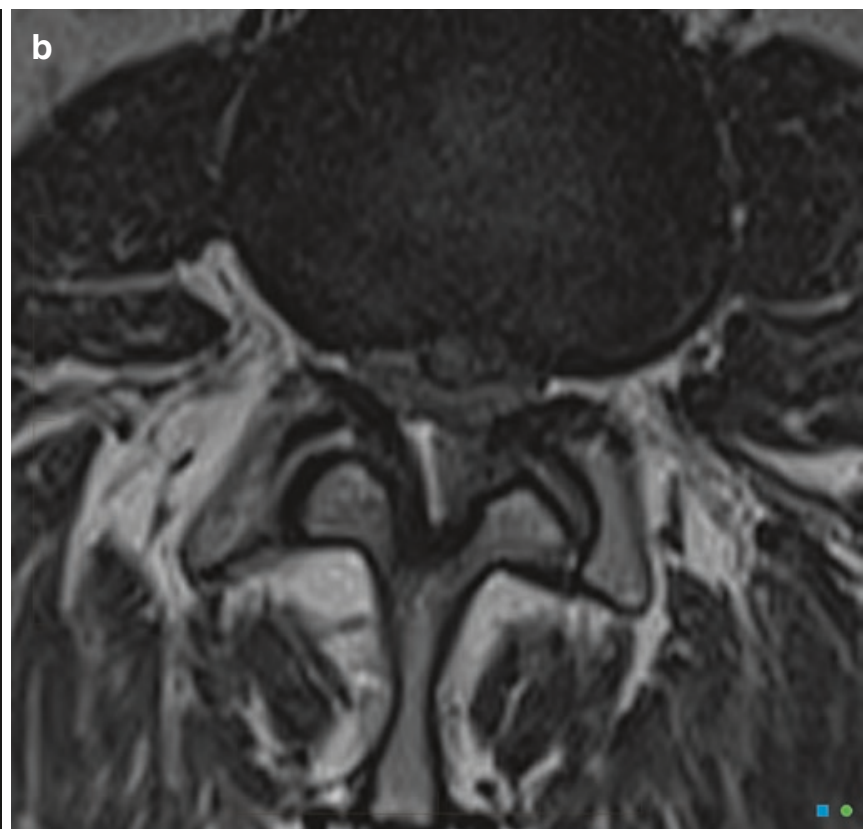

from the spinal canal or the nerves of the cauda equina are squeezed together 
ture. It is without doubt the best technique for assessing nerve root compression. Perhaps the only weakness is when there is metal implanted surgically and despite newer metal artefact suppression techniques, the image will be distorted at the level of the metal. MRI has particular strength in assessing for bone oedema and haemorrhage in acute subtle fractures.

\subsubsection{Isotope Bone Scintigraphy and White Cell Scintigraphy}

These techniques have a particular use in patients where pain is unexplained by MRI or when there are so many abnormalities found using MRI that it is uncertain which is the origin. Increased metabolic activity or white cell accumulation at a particular site may point to this as the most likely origin of symptoms. The radiation dose and the nonspecificity of the techniques are sufficient to make this a second-line test rather than a primary investigation.

\subsubsection{Interventional Techniques}

In the assessment of patients with degenerative arthropathy, disc degeneration and nerve root irritation, local anaesthetic blocks optionally with the use of steroids may allow the examiner to determine the exact origin of pain. 12 hours of complete relief after a $1 \mathrm{~mL}$ injection of bupivacaine clinches the origin of the symptoms. Therapeutic interventions include anaesthetic and steroid blocks to joints or nerve roots, radiofrequency rhizolysis to denervate facet joints, laser and radiofrequency disc ablation for disc-generated pain and percutaneous annuloplasty for symptomatic disc protrusion and are all potential techniques in the spine. Sacroiliac joint injections and sacroiliac ligament injections may help to determine whether the joint or the surrounding tissues are the origin of pain. Radiofrequency denervation of the sacroiliac joint may be useful [14] (Fig. 14.10).

\section{Key Point}

- Whilst MRI is the primary investigation for many of those with spine disorders, it is not without its limitations; conventional radiographs, CT and bone scintigraphy have an important role as secondary investigations.

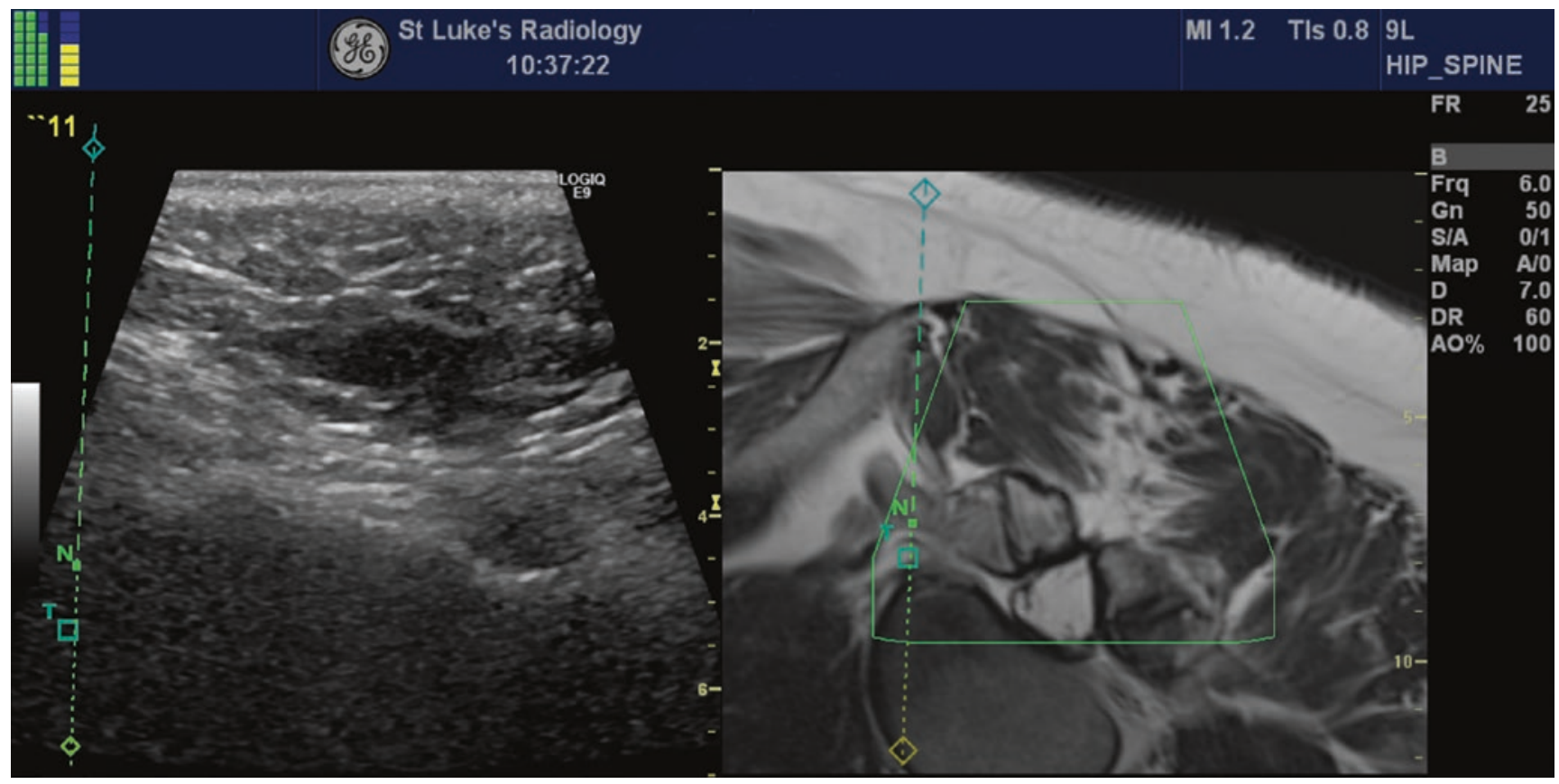

Fig. 14.10 Fusion imaging-guided nerve root block 


\subsection{Inflammatory Disorders of the Spine}

Noninfective inflammation of the spine is a frequent cause of back pain. It may be caused by spondyloarthropathy (SpA), rheumatoid arthritis (RA) or other less frequent inflammatory diseases. Inflammatory spondyloarthropathy is often overlooked on routine reporting. The modern management of inflammatory properties depends on early diagnosis and treatment with anti-inflammatory drugs or biologic agents, and a careful imaging strategy is important to avoid misdiagnosis and delayed treatment.

Magnetic resonance imaging (MRI) is the best method of assessing inflammatory disorders of the spine because it has high sensitivity and specificity. Conventional radiography, computed tomography (CT) and scintigraphy can also be employed to assess some of the inflammatory sites within both the axial skeleton and have specific secondary indications.

Key Point

- Early imaging is key to determining effective treatment.

\subsubsection{Imaging Methods in Evaluation of Inflammatory Spine}

\subsubsection{Conventional Radiographs}

Conventional radiographs of the spine will demonstrate bone morphology, calcification, ossification, large erosions and spine alignment. However, all of those are late-stage findings of inflammatory disorders. Consequently, conventional radiographs have limited value in the initial diagnostic assessment. It is useful for the clinical follow-up to detect complications and record progress.

\subsubsection{Computed Tomography (CT)}

CT will not demonstrate inflammatory changes at the early stage when they would be best treated. Joint erosions, subchondral sclerosis and bony ankylosis are easily detected by CT examination which for this purpose is far superior to conventional radiographs. CT is the ideal method of assessing complex fractures, bone lesions, spinal canal stenosis and articular joints osteoarthritis (facet joints, costovertebral joints, manubriosternal joints).

\subsubsection{Magnetic Resonance Imaging (MRI)}

MRI is very effective in the early diagnosis of SpA, RA and also any inflammation of the spine. MRI-specific protocols for inflammation should be employed, using fluid-sensitive sequences such as STIR (short Tau inversion recovery) or T2 weighted with fat suppression techniques in sagittal and coronal planes, with adequate signal-to-noise ratio and adequate field homogeneity. In most high field scanners, a STIR sequence demonstrates better performance over a T2 weighted sequence. Areas of increased signal on those fluidsensitive sequences correlate with oedema or vascularized fibrous tissue, termed bone marrow oedema (BME).

$\mathrm{BME}$ refers to nonspecific MR signal increase of the marrow cavity on fluid-sensitive sequences. Depending on cause, histological examination may demonstrate interstitial haemorrhage, organizing granulation tissue, necrosis, fibrosis, cellular infiltrate or reparative microcallus $[15,16]$. Alternative terms include BME pattern, BME-like signal and oedemalike marrow signal. On T1-weighted images, marrow fat can be effaced but not entirely replaced, differentiating BME from malignancy. BME has a similar MR appearance in traumatic, degenerative, inflammatory, neoplastic, ischaemic and idiopathic conditions. In RA, SpA, SAPHO (synovitis, acne, pustulosis, hyperostosis, osteitis) and CRMO (chronic recurrent multifocal osteomyelitis), BME indicates inflammation (osteitis, enthesitis).

MRI may also demonstrate sites of old bone marrow inflammation, when the bone marrow turns into yellow marrow with high signal intensity on T1-weighted sequences, a finding encountered with frequency at anterior vertebral corners or at sacroiliac joints.

MRI may fail to demonstrate ossification and fusion of other spinal elements such as the apophyseal joints, paraspinal ligaments and interspinous ligaments, and it may not fully identify syndesmophytes. A further weakness of MRI is that implanted metal will distort the image. This effect may be minimized with utilizing novel MRI sequences with artefact reduction capability.

\subsubsection{Isotope Bone Scintigraphy and White Cell Scintigraphy and FDG PET/CT}

FDG (fluorodeoxyglucose) PET (positron emission tomography)/CT (computed tomography) and other methods of scintigraphy have a specific use in patients where pain is unexplained by MRI or when there are so many abnormalities using MRI that it is uncertain which is important. The increased metabolic activity or white cell accumulation at a 
particular site suggests that this is the most likely origin of symptoms. The radiation dose and the non-specificity of the techniques are sufficient to make this a second line test rather than a primary investigation.

\subsubsection{Inflammatory Diseases of the Spine}

\subsubsection{Rheumatoid Arthritis (RA)}

Rheumatoid arthritis affects the cervical spine primarily. When that occurs, patients frequently develop anterior atlantoaxial subluxation. Abnormalities in the thoracic and lumbar spine are relatively rare. Disease onset is most frequent between the ages of 35 and 50 years [17].

\section{Imaging Findings of RA}

Features of spinal involvement in rheumatoid arthritis include erosive synovitis, ligamentous subluxation and osteopenia. Synovitis can be well detected with MRI as it demonstrates increased joint fluid at synovial joints (facet joints); in addition inflammatory osteitis may be observed when BME is present. Erosions are better detected with CT than by MRI or conventional radiography $[18,19]$.

\subsubsection{Spondyloarthropathies (SpA)}

Spondyloarthropathies are considered autoimmune disease mediated by antigen/antibody reactions that usually take place at the attachment of ligaments to bone (enthesitis). The term enthesitis is reserved for rheumatological disorders that cause primary entheseal and osseous inflammation which are hallmarks of spondyloarthropathy [19]. In the spine, this process occurs where the collagen of the ligaments (especially the longitudinal ligaments) or intervertebral disc annulus enters bone directly. The cause of the inflammatory process is the generation of cytokines which results in oedema, bone erosion, disorganization of bone and ligament structure which in turn promotes a reactive osteitis and eventually ossification of the ligaments starting at the enthesis interface.

SpA include ankylosing spondylitis, psoriatic arthritis, reactive arthritis, enteropathic $\mathrm{SpA}$ and undifferentiated $\mathrm{SpA}$. The seronegative spondyloarthropathies can be further categorized based on the imaging findings correlated with the clinical features and laboratory findings. Early diagnosis is key as disease-modifying drugs and physical therapy should be commenced as early as possible.

\subsubsection{Ankylosing Spondylitis (AS)}

This inflammatory disorder is the most frequent seronegative inflammatory spinal disease in adults and the prototype for other members of the spondyloarthropathies [20]. The prevalence of this disorder is estimated at $0.1-0.2 \%$ in the general population. Ankylosing spondylitis primarily affects young males, with a male-to-female ratio between $4: 1$ and 10:1. The mean age of presentation is 26 years. The HLA-B27 gene (human leukocyte antigen B27) is present in $95 \%$ of patients, but only a small percentage of all individuals positive for HLA-B27 actually develop the disorder [21].

AS typically begins in the sacroiliac joints and then affects the thoracolumbar and lumbosacral regions of the spine. Eventually, the spine becomes completely rigid, with loss of its normal curvatures and movement. Untreated, the disease will progress to bony ankylosis of joints which renders the spine at risk of transverse fractures following relatively minor injury. In the late stage, squaring of the vertebral bodies is a characteristic feature of AS and is caused by a combination of corner erosions and periosteal new bone formation along the anterior aspect of the vertebral body. Further inflammation is associated with formation of syndesmophytes: ossifications of the outer fibres of the annulus fibrosus that form bony bridges between the inflamed corners of adjacent vertebrae. Ossification also occurs within the fibres of the adjacent paravertebral connective tissue and of the posterior interspinous ligaments. Complete fusion of the vertebral bodies by syndesmophytes and related ossification produces the "bamboo spine" where the bone mimics a bamboo cane with segments of thickening. Fractures in AS usually occur at the thoracolumbar and cervicothoracic junctions where the biomechanical load is greatest. Spinal fractures are characteristically transverse (i.e., horizontal) rather than vertical or oblique and are called "chalk stick" or "banana" fractures. This is because these items will break transversely if bent.

There is considerable confusion in the literature about the difference between pseudarthrosis and discovertebral lesions. The Andersson lesion occurs where there is inflammatory involvement of the intervertebral disc; this may resemble disc infection. Excessive movement may occur at this level of destruction. This condition may develop spontaneously without any trauma. An advanced Andersson lesion is sometimes termed pseudarthrosis. However, pseudarthrosis may also occur when there has been a transverse fracture through bone and failure to unite. There are therefore three different groups of pseudarthrosis:

1. Localized lesions that always are of inflammatory origin and affect the disc space.

2. Extensive lesions without a fractured posterior element due to a combination of inflammatory and mechanical factors; they always are trans-discal and are associated with unfused facet joints.

3. Extensive lesions with fractured posterior elements resulting from mechanical fractures which may be transdiscal or trans-vertebral. 
On balance, the term pseudarthrosis is best reserved for ununited unstable transverse fractures of bone, whilst Andersson lesions (discovertebral lesions) are potentially unstable disc spaces that do not involve the osseous skeleton.

\subsubsection{Psoriatic Arthritis}

This is a unique inflammatory arthritis associated with psoriasis. Among patients with psoriasis, the prevalence of inflammatory arthritis varies from $6 \%$ to $42 \%$ [22].

\subsubsection{Reactive Arthritis (Reiter Syndrome)}

This is an acute non-purulent seronegative arthritis that complicates an infection elsewhere in the body and can also be associated with the HLA-B27, but this gene is not always present in affected individuals. The peak onset is between 15 and 35 years. An estimated 1-3\% of all patients with a nonspecific urethritis develop an episode of arthritis. Chronic arthritis or sacroiliitis occurs in $15-30 \%$ of patients [23]. The knee is most commonly affected followed by the ankle, small peripheral joints and sacroiliac joints. Some patients develop enthesitis (especially the calcaneus at attachment sites of the Achilles tendon and plantar fascia), dactylitis, urethritis and conjunctivitis. Diagnosis is based on clinical and laboratory findings. In chronic reactive arthritis ( $>6$ months), radiographs may show signs of inflammatory arthritis or sacroiliitis. In earlier stages, MR and US (ultrasound) demonstrate nonspecific synovitis, asymmetric sacroiliitis and calcaneal enthesitis.

\subsubsection{Enteropathic SpA}

This condition occurs in patients with inflammatory bowel diseases (IBDs) and other gastrointestinal diseases, such as Whipple's disease (WD), celiac disease (CD) and intestinal bypass surgery [24].

\subsubsection{Undifferentiated SpA}

This term used to describe symptoms and signs of spondylitis in someone who does not meet the criteria for a definitive diagnosis of AS or a related disease.

\subsubsection{Juvenile SpA}

A term used to classify both differentiated and undifferentiated forms of SpA disorders that begin at 16 years of age or younger.

\subsubsection{Imaging Findings of Spondyloarthropathy}

\subsubsection{Spondylitis}

Inflammation of the vertebral body (spondylitis) is due to enthesitis at the insertion of the annulus fibrosus on the ring apophysis of the vertebral end plate predominantly at the anterior attachment where there are usually the more florid manifestations. Erosions with reactive sclerosis in the vertebral corners are seen radiographically and have been referred to as "Romanus lesions" when viewed as erosions, and "shiny corners" when the erosion is associated with sclerosis. The Romanus lesions resolve by producing syndesmophytes (ossification of the outer fibres of the annulus fibrosus). When it progresses to involve multiple segments, it can produce the characteristic "bamboo spine". The same inflammatory process results in ossification of the longitudinal ligaments which insert onto the vertebral bodies producing squaring of the vertebral body as the fusion progresses.

MRI is the most sensitive diagnostic tool for the identification of discovertebral inflammatory disease when BME is present. The Romanus lesions are identified on the sagittal sequences and characterized by a triangular pattern of BME at the corners of the vertebral end plates highlighted by low T1 signal and high T2 fat-sat and STIR sequence appearance. The small erosion may be overlooked when compared with the areas of BME. After the acute Romanus lesion phase subsides, the chronic lesions are identified by a fatty marrow replacement at the sites of enthesis inflammation within the vertebral bodies, highlighted by a high $\mathrm{T} 1$ signal and a low signal on STIR and T2-weighted fat-saturated sequences. The intervertebral disc in cases of long-term spinal fusion can also undergo changes with high T1 signal, due to the presence of calcification or ossification. Contrast agent-enhanced MRI studies and diffusion-weighted MRI sequences can be useful in cases where the STIR images are equivocal but is usually unnecessary when STIR or T2-weighted fat saturation are well performed [25] (Fig. 14.11).

\subsubsection{Spondylodiscitis (Aseptic)}

\section{Primary Spondylodiscitis (Andersson Type A Lesions)}

This disorder resembles Schmorl's nodes exhibiting a rim of oedema within the vertebral body, a focal end plate defect 

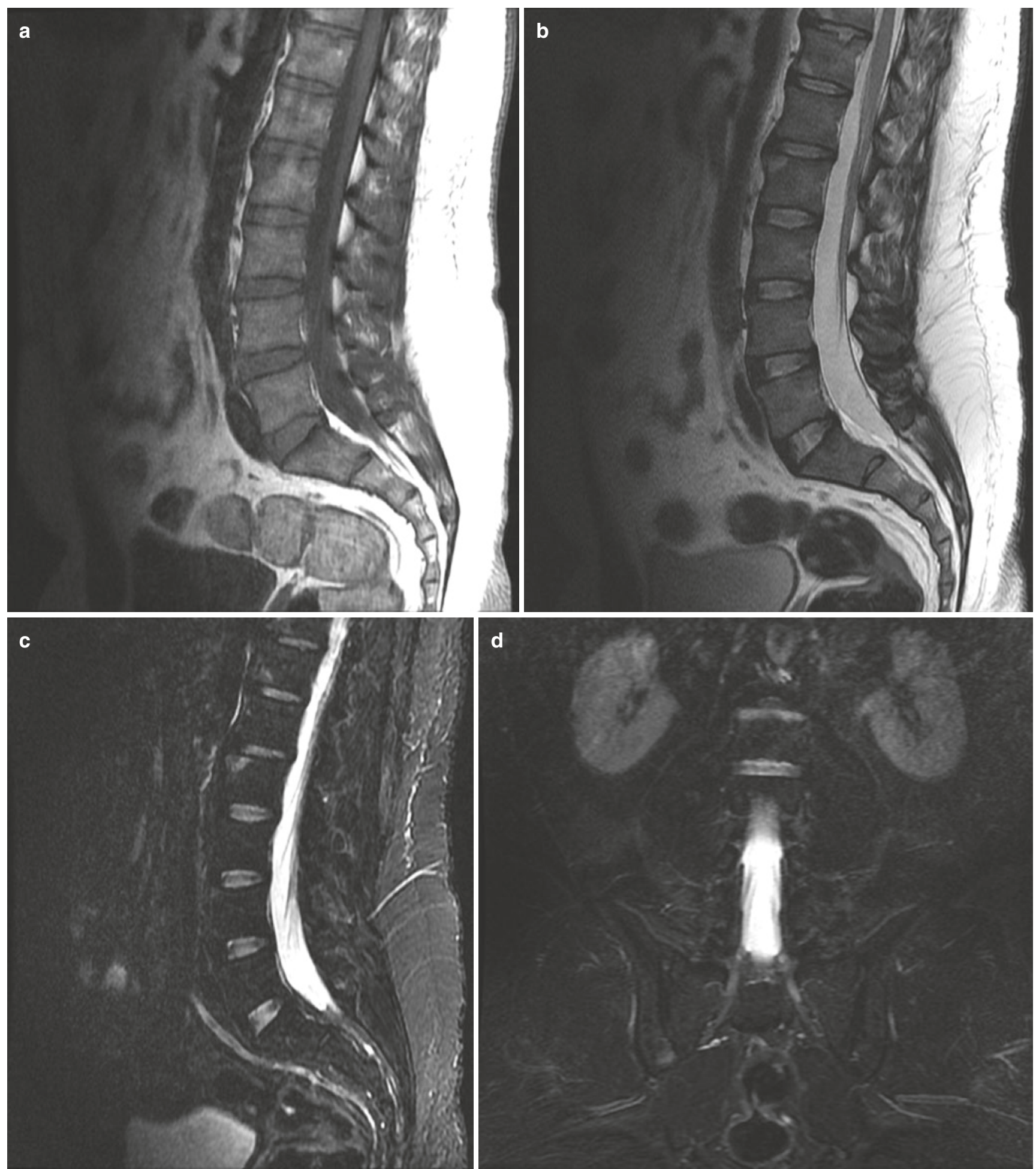

Fig. 14.11 (a) Sagittal T1-weighted MR image demonstrating Romanus lesions at L2 and T12 vertebral bodies, with low and high signal, respectively. (b) Sagittal T2-weighted MR image demonstrating

strating high signal of Romanus lesions with active inflammation. (d) Coronal STIR sequence demonstrating signs of bilateral asymmetric active inflammatory sacroiliitis both high signal Romanus lesions. (c) STIR sagittal MR image demon- 
and enhancement of the marrow oedema. Anderson type A lesions are usually a sign of early discovertebral involvement with a stable spinal status.

\section{Secondary Spondylodiscitis (Andersson Type B Lesion)}

This condition shows more extensive and florid discovertebral disease and destruction. These are particularly well demonstrated on CT and MRI. The degree of vertebral destruction is usually mild, but there is often extensive bony oedema and bony sclerosis, and in long established cases the end plates can be completely destroyed on both sides of the intervertebral disc. In Andersson type B lesions, the spine is unstable at the site of involvement due to increased mobility. This increased mobility could be at a level between fused segments or be associated with deficiency of the posterior elements where there is a pseudoarthrosis due to a fracture [25] (see above Hardy pseudarthrosis versus discovertebral disease) (Fig. 14.12).

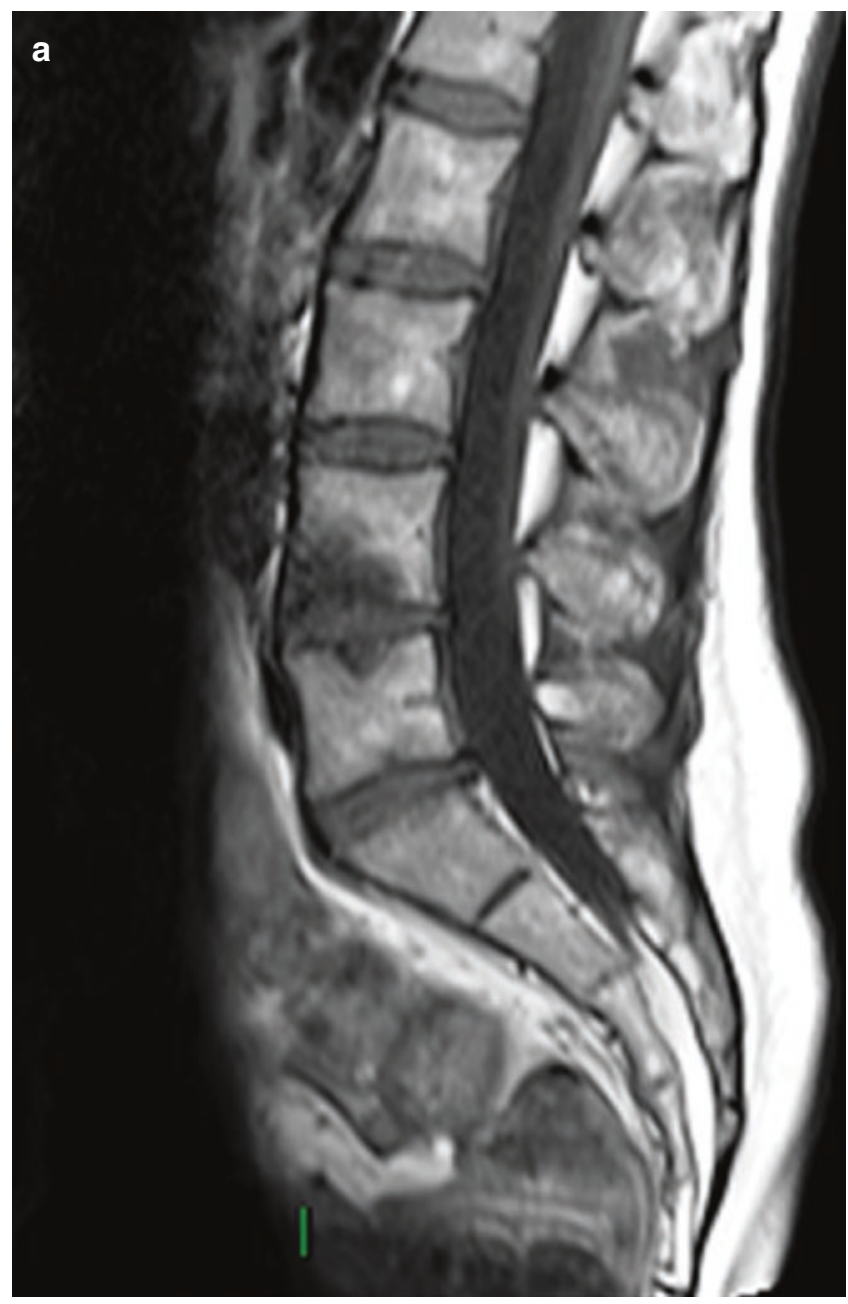

\section{Costovertebritis}

This is a very typical finding of spondyloarthropathy usually starting in the lower thoracic spine. The inflammatory process with soft tissue oedema and bone oedema is seen in the costovertebral joints best seen on sagittal images. These are often overlooked when reporting MRI examinations because the slices showing the disease process are at the margin of the vertebral column.

\section{Sacroiliitis}

Sacroiliitis is a hallmark of all spondyloarthropathies. In AS and enteropathic SpA, it is usually bilateral and symmetrical, whilst in psoriatic spondyloarthropathy and reactive arthritis, it may be bilateral or unilateral. Involvement of the axial skeleton is not unusual in the absence of sacroiliitis, especially with the use of high-quality fluid-sensitive sagittal STIR and T2-weighted fat saturation sequences.

Conventional radiography has low sensitivity and relatively high false-negative rate in early disease. Early inflam-

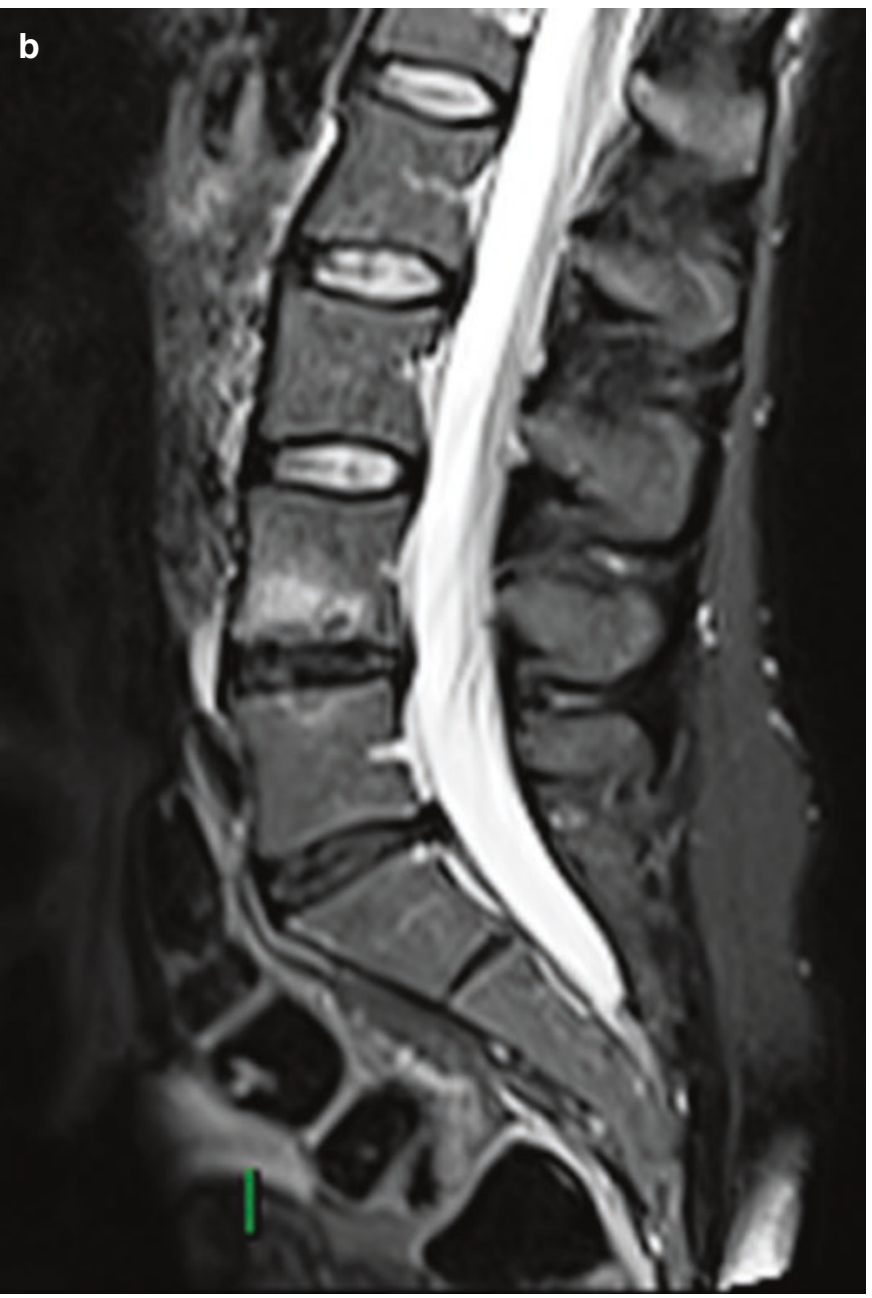

Fig. 14.12 (a) Sagittal T1-weighted MR image demonstrating Andersson lesions at L4-L5 level, with low signal. (b) Sagittal STIR demonstrating BME of Anderson inflammatory spondylitis 
matory sacroiliitis is very difficult to access. Sclerosis of the subchondral bone on either side of the joint is fairly diagnostic in established disease and is more pronounced on the iliac side unlike infection of the sacroiliac joint which affects both sides equally. In established disease, the sacroiliac joint may also exhibit loss of sharpness due to ossification across the joint leading to ankylosis. The relatively late development of radiographic changes in ankylosing spondylitis is one reason for delay in diagnosis.

MR imaging has revolutionized the early diagnosis of sacroiliitis. This is primarily dependent on osteitis, an important feature of ankylosing spondylitis, which produces BME which is easily detected upon sensitive sequences such as T2-weighted sequences with fat suppression or the STIR sequence. The T1-weighted spin-echo sequences are better at depicting articular erosions. The degree of the oedema may vary ranging from florid, fairly extensive areas of periarticular oedema to more focal and localized zones of oedema paralleling the joint line. It is usually the inferior iliac portion of the joint that is involved in the early stages of sacroiliac inflammatory change as this is the sign of your component of the sacroiliac joint early commitment. Contrast agent enhancement can be useful if the oedema-sensitive sequence (STIR) is equivocal. CT is preferred for the detection of very early erosions of the sacroiliac joints and their early ankylosis.

\section{Key Point}

- Comprehensive imaging is an essential element of the diagnosis, but the conclusion will depend on the combination of imaging, clinical history and laboratory investigations.

\subsubsection{Complications of Inflammatory Joint Disease}

The most important spinal complications in ankylosing spondylitis are osteoporosis, fracture, instability, cauda equina syndrome and spinal stenosis.

Osteoporosis increases in prevalence directly with increased patient age, increased severity of spinal involvement, increased disease duration and peripheral arthritis. Bone fragility associated with osteoporosis obviously increases the chances of vertebral compression fractures, posterior element fractures, pseudoarthrosis and unstable fractures from relatively minor trauma. The treatment of inflammatory arthropathy may accelerate osteoporosis.

Fractures of the cervical spine may occur after a minor fall or injury to the head and neck. Typically, the conventional radiographs show a chalk stick type of break either through the disc or the vertebral body anteriorly and horizontally through the posterior fused elements. A common spinal location for fracture is the thoracolumbar and cervicothoracic and lastly the lumbosacral junction. Any ankylosing spondylitis patient suffering minor trauma who complains of pain should have advanced imaging preferably by $\mathrm{CT}$, as this will be most sensitive in detecting what are often very subtle but grossly unstable injuries. There should be a very low threshold for performing CT examinations with ankylosing conditions of the spine even after very mild trauma (Fig. 14.13).

Cauda equina syndrome is a rare but specific complication following long-standing ankylosing spondylitis. It invariably occurs in a fused spine and is most common in the lumbar region. Dural ectasia is common, resulting in erosions of primarily the posterior neural arch. This is best assessed by CT or MRI. MRI will show enlargement of the spinal canal with arachnoid diverticulae, erosion of the laminae and adherent nerve roots.

Spinal stenosis can be observed with ossification of the longitudinal ligament and the ligamentum flavum. Neurological deficit in patients with AS could have a number of causes, but $\mathrm{C} 1-\mathrm{C} 2$ subluxation, fracture, pseudoarthrosis, ligamentous ossification and cauda equina syndrome are the most common.

\section{Key Point}

- In ankylosing spondyloarthropathy, fractures may occur with minor trauma and are frequently severely unstable. Early cross-sectional imaging is essential in these cases which may be very difficult to interpret.

\subsubsection{Other Inflammatory Diseases That Could Affect the Spine}

\subsubsection{SAPHO}

SAPHO syndrome represents a rare inflammatory disorder involving the bone, joints and skin. The acronym refers to synovitis, acne, pustulosis, hyperostosis and osteitis. Although the aetiology is probably autoimmune, Cutibacterium acnes infection may activate the process in some cases. Cultures are usually negative, and biopsy shows sterile, nonspecific osteitis without sequestrum formation. SAPHO has been associated with axial spondyloarthropathy and CRMO, a childhood variant of chronic nonbacterial osteomyelitis (CNO). In adults, commonly involved sites include the sternocostoclavicular complex followed by the spine and sacroiliac joints. Radiographs and CT demonstrate erosive costoclavicular ankylosis, osteosclerosis and thick periosteal reaction of the clavicle and first rib. Bone scintigraphy shows the "bull's head sign" (avid uptake of 


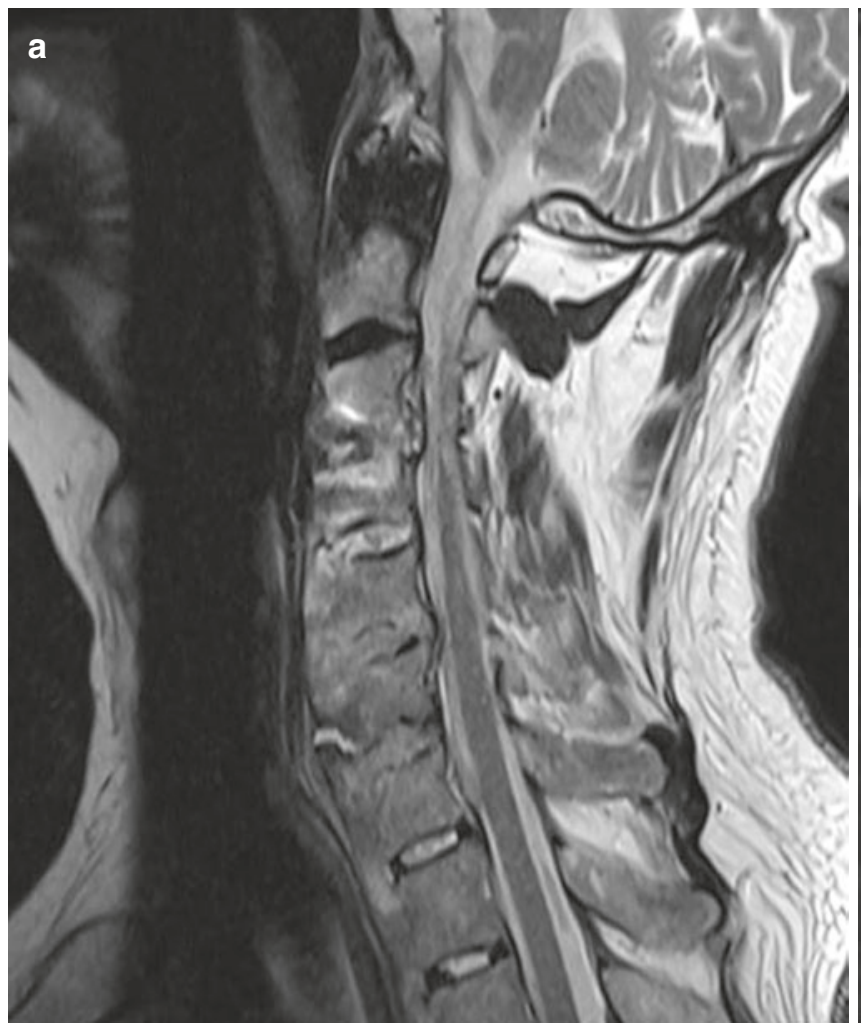

Fig. 14.13 (a) Sagittal T2-weighted MR image of cervical spine demonstrating multiple levels of bone fusion by AS from $\mathrm{C} 3$ to $\mathrm{C} 6$ with ossification demonstrated as low signal at disc space and spinal stenosis at C3-C4. (b) STIR sagittal MR image demonstrating myelopathy at

99mTc-methylene diphosphonate by the sternomanubrial and bilateral sternocostoclavicular regions). In polyostotic disease, bone scintigraphy, PET and whole-body MRI are valuable for skeletal screening to identify sites of active osteitis. MRI can demonstrate early osteitis, synovitis, enthesitis, spondylodiscitis and sacroiliitis. In children and adolescents, long bone metaphases are commonly involved. Differential diagnosis includes osteomyelitis and malignancy.

\subsubsection{Sarcoidosis}

Sarcoidosis is a systemic granulomatous disease of unknown cause that has been shown to affect nearly every body system. Osseous involvement occurs in 1-13\% of patients, typically in the small tubular bones of the hands and feet. Sarcoidosis is usually treated with corticosteroid [26].

\subsubsection{Crystal Deposition Disorders}

In crystal deposition disease, collections of crystal may be found in musculoskeletal tissues in asymptomatic individuals. In others these deposits may produce severe pain and profound symptoms. This systemic disease associated with

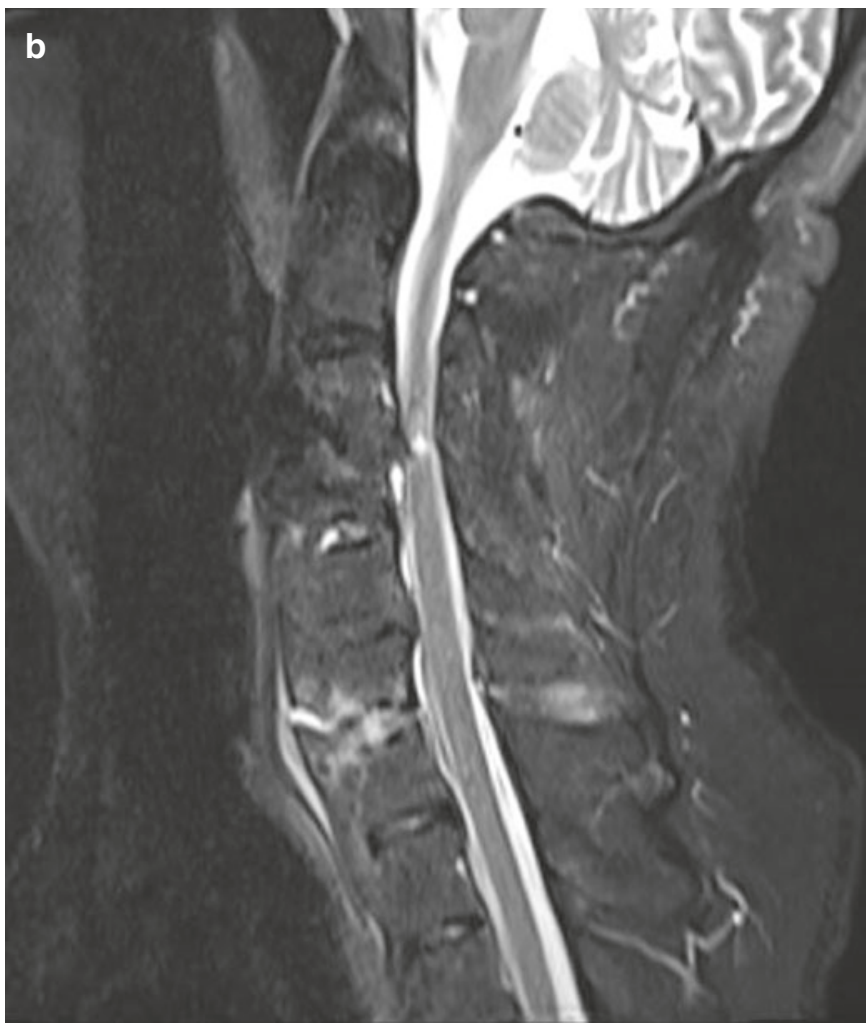

C3-C4 level and high signal of active inflammation (Anderson lesion) of spondylitis process, as well as thick and increase signal of the anterior longitudinal ligament

some crystal disorders may be occult, and musculoskeletal deposits may be the presenting feature.

The common crystal-related deposition disorders are calcium pyrophosphate dihydrate (CPPD), hydroxyapatite and monosodium urate deposition. MRI and CT examination features include (a) location (discs, hyaline cartilage and fibrocartilage, ligaments, bone, synovial membrane), (b) morphology (size, soft tissue signal characteristics), (c) surrounding tissue signal change and (d) arthritis (bone, synovial membrane). There are different patterns with individual diseases.

CPPD crystal deposit can be found with a high frequency in older people in the discs, hyaline cartilage and ligaments. Calcification in the spinal ligaments especially around the odontoid process is common. Focal calcifications forming conglomerates around joints like tophaceous gout may be also seen. It is often seen in women $>80$ years old ("disease of octogenarians"). Intervertebral disc CPPD crystal deposits can be found in asymptomatic individuals but in some more rare cases can cause destructive disc disease. 
Calcium hydroxyapatite crystals (HA) deposits also can be found in the spine in asymptomatic individuals, often intradiscal. At high calcium concentrations (above 30-40\%), MRI susceptibility defects and decreases in proton density dominate, leading to signal intensity reduction. However, T1 shortening defects resulting in hyperintensity on T1-weighted images may also be present. They have been attributed to surface interaction of protons with calcified tissue. At lower concentrations of calcium, T1 shortening effect dominates, resulting in isointensity or even hyperintensity. Gradient echo sequences best show these calcific foci, but the gold standard confirmation is made with CT. The deposition of HA may mimic an acute inflammation (Figs. 14.14 and 14.15).
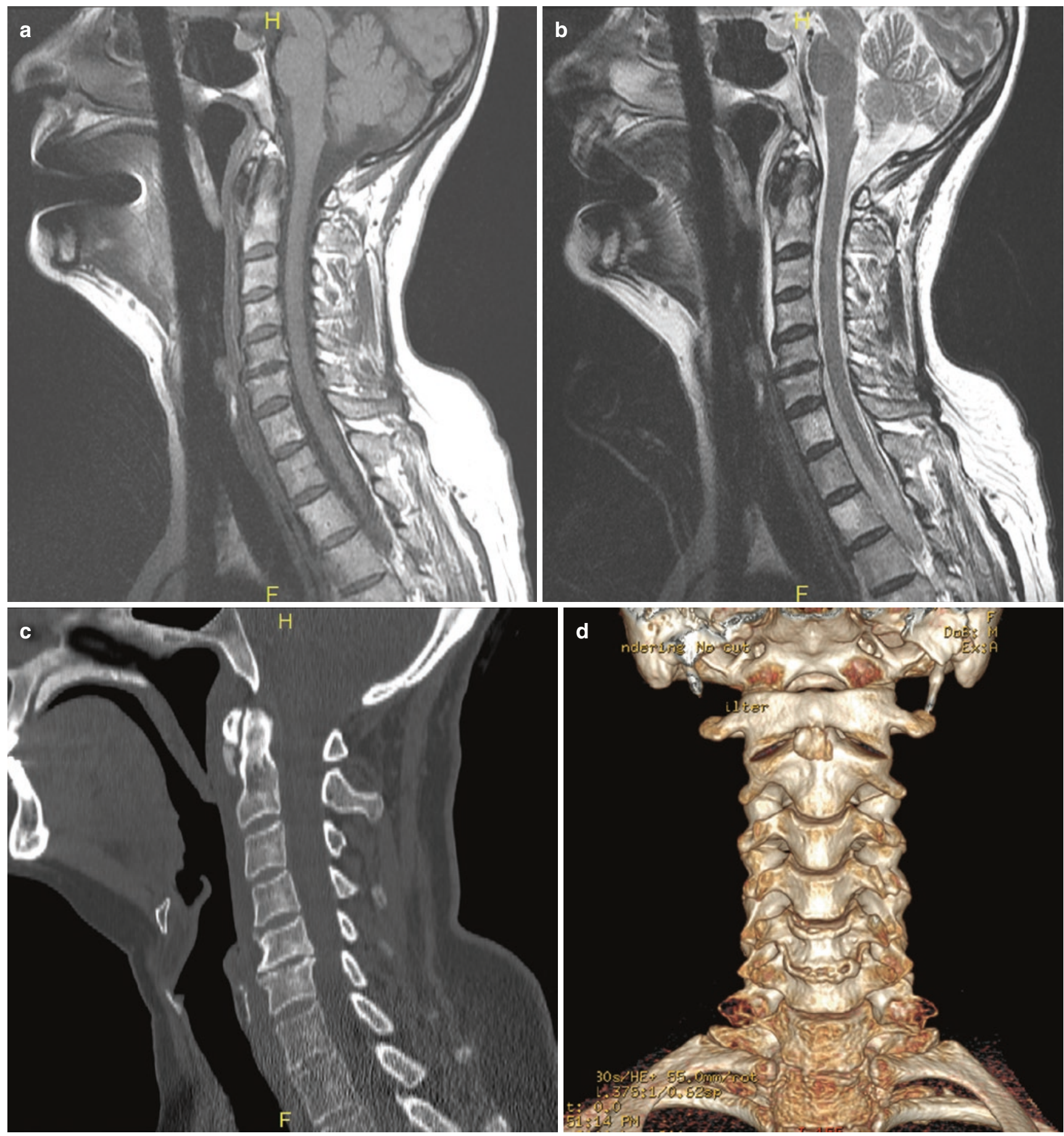

Fig. 14.14 Longus colli muscle insertion calcification by hydroxyapatite crystal deposition demonstrated with (a) sagittal T1-weighted MR image, (b) sagittal T2-weighted MR image, (c) CT sagittal CT reformat and (d) CT 3D reconstruction 

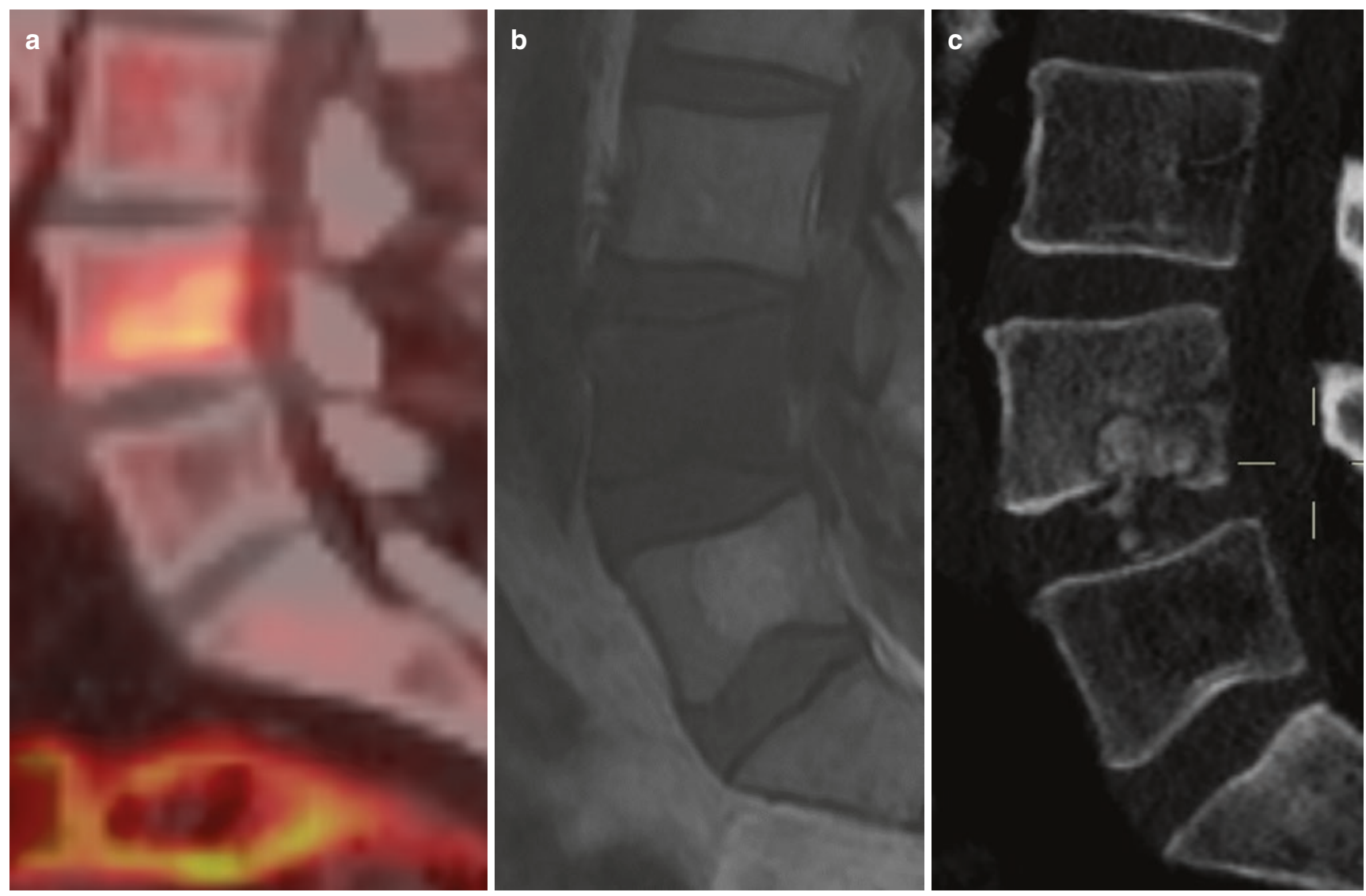

Fig. 14.15 Migration of hydroxyapatite crystal deposition into vertebral body of L4 causing spondylitis demonstrated with (a) FDG PET/CT, (b) sagittal T1-weighted MR image and (c) CT sagittal CT reformat

Monosodium urate monohydrate crystals or uric acid deposits into tissues may cause gout. It happens when hyperuricemia is above $6-7 \mathrm{mg} / \mathrm{dL}$. Gout is the most common inflammatory arthritis in men over the age of 30 year but is uncommon in the spine. The radiographic findings of gout do not occur until the disease has been present for at least 6 or more years. In the acute phase, MRI findings are joint effusion and synovial thickening although these are nonspecific signs. Gouty tophi can be found in rare cases in the spine arising from facet joints. They are mostly of low or intermediate signal intensity on T1-weighted images with variable gadolinium enhancement. The imaging findings of tophi on T2-weighted images are variable. Calcification, fibrosis or hemosiderin can occur within the tophi. With dual source computed tomography, the uric acid deposits can be differentiated with considerable accuracy and high imaging contrast.

\section{Key Point}

- Inflammatory disease of the spine is not confined to ankylosing spondylitis.

\subsection{Concluding Remarks}

Degenerative and inflammatory disease of the spine may overlap in their signs and symptoms. There is a wide range of disorders, and radiologists must have a comprehensive knowledge of the presentation of these diseases. Imaging is now key in the critical stages of management, for example, in assessing spinal stability and helping to determine treatment with powerful anti-inflammatory drugs. MRI is the primary investigation in many spine disorders, but conventional radiographs and $\mathrm{CT}$ are more important first-line techniques in trauma. There will be occasions when a combination of imaging is required to make a precise and safe diagnosis.

\section{Take Home Message}

- Those who undertake reporting of spinal disorders using any imaging technique must have a comprehensive knowledge of disease processes, their symptoms and imaging signs. 


\section{References}

1. Adams MA, Bogduk N, Burton K, Dolan P. The biomechanics of back pain. 3rd ed. Edinburgh: Elsevier Churchill Livingstone; 2013.

2. Bogduk N, Aprill C. On the nature of neck pain, discography and cervical zygapophysial joint blocks. Pain. 1993;54:213-7.

3. Aprill C, Bogduk N. High-intensity zone: a diagnostic sign of painful lumbar disc on magnetic resonance imaging. Br J Radiol. 1992:65:361-9.

4. Jha SC, Higashino K, Sakai T, et al. Clinical significance of highintensity zone for discogenic low back pain: a review. J Med Investig. 2016;63:1-7.

5. Goupille P, Mulleman D, Valat JP. Radiculopathy associated with disc herniation. Ann Rheum Dis. 2006;65:141-3.

6. An HS, Masuda K, Inoue N. Intervertebral disc degeneration: biological and biomechanical factors. J Orthop Sci. 2006;11: 541-52.

7. Sebro R, O'Brien L, Torriani M, Bredella MA. Assessment of trunk muscle density using $\mathrm{CT}$ and its association with degenerative disc and facet joint disease of the lumbar spine. Skelet Radiol. 2016;45:1221-6.

8. Scheuermann HW. The classic: kyphosis dorsalis juvenilis. Clin Orthop Relat Res. 1977;128:5.

9. Zaidman AM, Zaidman MN, Strokova EL, et al. The mode of inheritance of Scheuermann's disease. BioMed Res Int. 2013;2013:973716.

10. Palazzo C, Sailhan F, Revel M. Scheuermann's disease: an update. Joint Bone Spine. 2014;81:209-14.

11. Maatta JH, MacGregor A, Karppinen J, Williams FM. The relationship between Modic changes and intervertebral disc degeneration. BMC Musculoskelet Disord. 2016;17:371.

12. Xu L, Chu B, Feng Y, Xu F, Zou YF. Modic changes in lumbar spine: prevalence and distribution patterns of end plate oedema and end plate sclerosis. Br J Radiol. 2016;89:20150650.

13. Machado GC, Ferreira PH, Yoo RI, et al. Surgical options for lumbar spinal stenosis. Cochrane Database Syst Rev. 2016;11: CD012421.
14. Pope TL, Bloem HL, Beltran J, Morrison WB, Wilson DJ. Musculoskeletal imaging. 2nd ed. Philadelphia, PA: Elsevier Saunders; 2015.

15. Hiryayi WA, Thiryayi SA, Freemont AJ. Histopathological perspective on bone marrow oedema, reactive bone change and haemorrhage. Eur J Radiol. 2008;67(1):62-7.

16. Zanetti M, Bruder E, Romero J, Hodler J. Bone marrow edema pattern in osteoarthritic knees: correlation between MR imaging and histologic findings. Radiology. 2000;215(3):835-40.

17. Calleja M, Hide G. Rheumatoid arthritis, spine. Available at http:// www.emedicine.com/radio/topic836.htm. Last updated 28 Mar 2006. Accessed 30 Jun 2007.

18. Hermann KG, Bollow M. Magnetic resonance imaging of the axial skeleton in rheumatoid disease. Best Pract Res Clin Rheumatol. 2004;18:881-907.

19. Selmi C, Gershwin ME. Diagnosis and classification of reactive arthritis. Autoimmun Rev. 2014;13:546-9.

20. Bollow M, Enzweiler C, Taupitz M, et al. Use of contrast enhanced magnetic resonance imaging to detect spinal inflammation in patients with spondyloarthritides. Clin Exp Rheumatol. 2002;20(Suppl 28):S167-74.

21. Peh W. Ankylosing spondylitis. Available at http://www.emedicine. com/radio/topic41.htm.

22. Gladman DD, Antoni C, Mease P, et al. Psoriatic arthritis: epidemiology, clinical features, course, and outcome. Ann Rheum Dis. 2005;64:14-7.

23. Scoggins T, Boyarsky I. Reactive arthritis. Available at http://www. emedicine.com/emerg/topic498.htm. Last updated 15 Feb 2007. Accessed 30 Jun 2007.

24. Peluso R, Di Minno MN, Iervolino S, et al. Enteropathic spondyloarthritis: from diagnosis to treatment. Clin Dev Immunol. 2013;2013:631408.

25. Wilson DJ, Casar-Pulliccino V. Spine degeneration and inflammation. In: Musculoskeletal disease (2017-2020). Cham: Springer; 2017.

26. Campbell SE, Reed CM, Liem T, et al. Radiologic-pathologic conference of Brooke Army Medical Center: vertebral and spinal cord sarcoidosis. AJR Am J Roentgenol. 2005;184:1686-7.

Open Access This chapter is licensed under the terms of the Creative Commons Attribution 4.0 International License (http://creativecommons. org/licenses/by/4.0/), which permits use, sharing, adaptation, distribution and reproduction in any medium or format, as long as you give appropriate credit to the original author(s) and the source, provide a link to the Creative Commons license and indicate if changes were made.

The images or other third party material in this chapter are included in the chapter's Creative Commons license, unless indicated otherwise in a credit line to the material. If material is not included in the chapter's Creative Commons license and your intended use is not permitted by statutory regulation or exceeds the permitted use, you will need to obtain permission directly from the copyright holder. 